\title{
FLUXO DE ATENDIMENTO ÀS MULHERES COM CÂNCER DE OVÁRIO ANTES DO ENCAMINHAMENTO AO NÍVEL TERCIÁRIO
}

\author{
Maria das Gracas Almeida Zaneti ${ }^{1}$ \\ Rosana Oliveira de Melo ${ }^{2}$ \\ 1. Bolsista PROBIC/UEFS, Graduanda em Enfermagem, Universidade Estadual de Feira de Santana, e-mail: \\ galmeida_fsa@hotmail.com \\ 2. Orientador, Departamento de Saúde, Universidade Estadual de Feira de Santana, e-mail: rosanaenfmelo@gmail.com
}

PALAVRAS-CHAVE: Saúde da Mulher, Fluxo de atendimento, Câncer de Ovário

\section{INTRODUÇÃO}

A mulher vivencia no seu ciclo de vida experiências relacionadas aos aspectos ginecológicos, sejam eles de caráter preventivo ou curativo. Dentre as diversas patologias ginecológicas que podem acometer a mulher têm-se com maior frequência, as infecções sexualmente transmissíveis (IST) e os cânceres ginecológicos.

Atualmente o câncer constitui-se um grave problema de saúde pública e este causa um grande impacto socioeconômico. Em 2016/2017, no Brasil, estima-se que ocorram 596 mil novos de câncer em consequência do aumento da expectativa de vida da população, da urbanização e da globalização (INCA, [2015]).

No que se refere as mulheres, têm-se as neoplasias ginecológicas, e de acordo a incidência, tem-se o câncer de mama em primeiro lugar, o câncer do colo do útero em segundo e ovário, em terceiro (INCA, 2015).

Apesar da neoplasia de ovário não ser tão incidente, apresenta alta taxa de letalidade. Após a confirmação diagnóstica, a expectativa de vida pode variar em até 5 anos devido ao diagnóstico tardio, tratamento ineficaz e principalmente, possíveis falhas no fluxo de atendimento dessas mulheres (DERCHAIN; FRANCO; SARAIN, 2009).

A prevalência do câncer de ovário incide em mulheres de cor branca, com idade superior a 50 anos, que apresentam sintomas comuns a outras doenças, tais como: inchaço, dor pélvica e sangramento. Em suma, por ser uma doença assintomática na maioria das vezes, dificulta o diagnóstico. Outro motivo que compromete o diagnóstico precoce é a localização profunda do ovário na pélvis, pois quando se torna palpável, já se encontra em estágio avançado (OLIVEIRA, 2014).

Devido à gravidade da doença é importante a conscientização da população sobre os fatores de risco, as formas de prevenção, a importância da realização periódica de exames como ultrassonografia, além do acompanhamento clínico, para assim chegar a um possível diagnóstico precoce; e isso se dará através de um atendimento adequado e resolutivo nos diversos níveis de atenção à saúde.

A atenção primária "prioriza ações de promoção, proteção e recuperação de saúde, de forma integral e continuada" (OLIVEIRA; PEREIRA, 2013, p. 159). A esta compete realizar ações de baixa complexidade, principalmente preventivas, e é nesse nível de atenção que deverão ser identificados os possíveis casos de câncer (ZEFERINO; COELHO, 2003). Por isso, é muito importante que os profissionais estejam capacitados para identificar e avaliar de forma clara e objetiva os casos suspeitos de câncer.

Diante o exposto, incorporam-se a falta de dados mais detalhados a respeito da doença e a escassez de conhecimento acerca do atendimento as mulheres na rede de atenção à saúde; faz-se necessário, ampliar o conhecimento dos profissionais e dos acadêmicos sobre a temática e oferecer à população em geral, informações sobre a doença.

O objetivo geral desse estudo foi analisar o fluxo de atendimento às mulheres com câncer de ovário antes do encaminhamento ao nível terciário. E como objetivo especifico descrever o atendimento às mulheres com câncer de ovário até a confirmação diagnóstica. 


\section{METODOLOGIA}

Estudo qualitativo, descritivo, realizado na Unidade de Alta Complexidade em Oncologia (UNACON), em Feira de Santana-BA. Vinculado ao projeto matriz intitulado "ATENÇÃO À SAÚDE DA MULHER NOS SERVIÇOS PÚBLICOS DO MUNICÍPIO DE FEIRA DE SANTANA - BA", aprovado pelo Comitê de Ética da UEFS sob o no 1.327.867 e pelo CONSEPE resolução $\mathrm{n}^{\circ}$ 008/2016. Participaram do estudo, 08 mulheres com diagnóstico de câncer de ovário, atendidas na unidade e que aceitaram participar da pesquisa. A mesma foi iniciada após a assinatura do Termo de Consentimento Livre e Esclarecido, e teve como critérios de inclusão: mulheres com idade igual ou superior a 18 anos e com câncer de ovário em tratamento na Unacon. Os critérios de exclusão foram mulheres com algum transtorno mental que impossibilite a compreensão e/ou colaboração com os objetivos da pesquisa. Para iniciar a pesquisa, procedeu-se um processo de ambientação para conhecer a equipe atuante, mediadores desse processo, assim como as participantes. A técnica e instrumento de coleta de dados utilizada foi a entrevista semi-estruturada, por meio de um roteiro e estas ocorreram em local privativo na própria unidade. Após anuência das participantes, as entrevistas foram gravadas, transcritas e analisadas. A técnica de análise utilizada foi a de conteúdo de Bardin com a leitura exaustiva dos dados, categorização e tratamento dos resultados. O estudo ocorreu em agosto de 2016 a maio de 2017 e respeitou-se os preceitos éticos da Resolução $466 / 2012$ e a identidade das participantes por meio de codinomes escolhido por estas.

\section{ANÁLISE E DISCUSSÃO DOS RESULTADOS}

Os dados desse estudo permitiram conhecer as características sociodemográficas e gineco-obstétricas das participantes, que são informações ligadas às características dessa neoplasia. Percebeu-se que as idades das mulheres variaram entre 41 a 81 anos, se aproximando da média estimada nos estudos. A maioria destas, eram pardas, casadas, católicas, pertencentes as microrregiões de saúde, ou seja, apenas três participantes eram de Feira de Santana; possuíam ensino fundamental incompleto, eram lavradoras e recebem até um salário mínimo.

De acordo as características gineco-obstétricas, as participantes possuíam um fluxo menstrual normal, 50\% destas, já estavam na menopausa e todas fizeram uso de anticoncepcional oral, o qual, a literatura destaca que, para esta neoplasia é um fator protetor. Todas tiveram filhos e amamentaram por um período de um mês e meio a três anos. Se percebeu também que $100 \%$ destas realizaram o exame de citologia oncótica e a ultrassom transvaginal, porém, apenas $50 \%$ faziam com frequência.

Quanto aos fatores de risco, as mulheres não possuíam histórico de câncer de ovário, de obesidade e não utilizaram terapia hormonal. Referente aos sinais e sintomas, $75 \%$ teve alteração gastrointestinal como aumento do volume abdominal ou palpação de massa pélvica e 50\% das pacientes cursaram com dor; observaram-se também alterações geniturinárias. Estes sintomas são inespecíficos, contribuindo para o diagnóstico tardio e são descritos na literatura (A. C. CAMARGO, 2014; OLIVEIRA; 2014; PITTA, 2013).

O tempo de descoberta da doença variou entre sete meses a cinco anos, segundo o relato das participantes. Nenhuma das pacientes conhecia o subtipo do câncer de ovário. Todos foram cânceres primários e destas, $25 \%$ estava em tratamento de recidiva, o que é comum. Neste sentido, Vaughan e outros (2011) corroboram com a assertiva de que, muitas mulheres respondem bem ao tratamento de primeira linha, no entanto, o tumor pode desenvolver resistência.

A trajetória das mulheres iniciou-se na busca aos serviços de saúde, com o aparecimento dos sinais e sintomas, e assim esperava-se que o diagnóstico ocorresse por exames de rotina. Percebeu-se ainda, que não há um percurso comum em seus discursos, e que o fluxo iniciou-se tanto na atenção básica $(\mathrm{AB})$, como na secundária ou terciária, porém 
outras mulheres precisaram buscar assistência não só nos serviços públicos, mas também, no sistema privado.

Ao considerar que o acesso aos serviços de saúde é direito de todos e o sistema público de saúde no Brasil tem princípios bases para a assistência, compreendeu-se que este encontra-se fragilizado. Destaca-se então a integralidade como um ponto importante na busca pelo acesso aos serviços. Nessa perspectiva, Texeira (2011, p. 6) acrescenta que "[...] Um modelo "integral", portanto, é aquele que dispõe de estabelecimentos, unidades de prestação de serviços, pessoal capacitado e recursos necessários, à produção de ações de saúde [...] (TEXEIRA, 2011, p. 6).

Espera-se que na $\mathrm{AB}$, as mulheres tenham um acompanhamento clínico por meio da anamnese detalhada e passem por rastreamento dos fatores de risco, pois é neste nível de atenção, que a assistência prestada tem como foco, a promoção e a prevenção da saúde. A partir da suspeita diagnóstica devem-se ser solicitados exames e se o resultado se aproximar do diagnóstico de câncer, a mulher deve ser assistida na atenção terciária. Nesse sentido, é importante manter a integralidade entre os níveis de atenção, para que os fluxos entre eles sejam de fácil acesso e ágeis (ASSIS et al., 2010).

Entretanto, observou-se que a assistência prestada, durante a suspeita diagnóstica até a sua confirmação apresentou falhas, que podem ser influenciadas pela inexistência de um fluxograma de atendimento pré-estabelecido e pelas fragilidades na comunicação entre os níveis de atenção. As mulheres precisaram, além de buscar outros meios como a assistências privada para dar resolubilidades, passar por momentos de incertezas com diagnósticos imprecisos, assim como vários encaminhamentos. Estas, ao buscarem os serviços de saúde passaram por diagnósticos incorretos e tratamento desnecessários até serem diagnosticadas com a neoplasia.

Quando a trajetória diagnóstica dessa mulher é marcada por vários desencontros, essa irá refletir diretamente no início do tratamento, comprometendo o prognóstico da doença. Além dos desafios para o diagnóstico, nota-se que, o uso da ultrassonografia transvaginal (USG), também é um desafio. Sabe-se que a USG apenas identifica anormalidades no órgão e que o diagnóstico ocorre apenas, após a biópsia, o que se enquadra, teoricamente, na trajetória terapêutica. Além da USG para identificar alterações ovarianas, tem-se também, os marcadores tumorais que são CA 125, HE4, CEA; destes, o mais utilizado é o CA 125.

Nessa pesquisa, compreendeu-se que houve uma divergência entre as suspeitas diagnósticas utilizando a USG transvaginal. Alguns profissionais que realizaram a USG identificaram as alterações, no entanto, outros, não identificaram. Esta ação reflete no diagnóstico tardio e prognóstico dessas mulheres. Diante da gravidade da doença e da importância do diagnóstico precoce, falhas como essas não deveriam ocorrer e vale repensar na fragilidade da assistência prestada e nas consequências da falta de um fluxograma de atendimento estabelecido, de investimentos para capacitações e/ou contratação de profissionais qualificados. Infelizmente, o investimento voltado à educação em saúde não é suficiente e o modelo de planos assistenciais, precisam ser repensados para assim, garantir uma assistência eficaz e que obtenha resultados positivos para esta neoplasia.

\section{CONSIDERAÇÕES FINAIS}

Este estudo revelou que as mulheres com câncer de ovário iniciaram suas trajetórias a partir do aparecimento de sinais e sintomas e em busca pelos serviços de saúde para resolubilidade. No entanto, vários foram os percursos até a confirmação diagnóstica. Percebeu-se que a maior parte dos diagnósticos ocorreram tardiamente, os sintomas apresentados foram inespecíficos e resultou em diagnósticos incorretos e procedimentos desnecessários. 
Sinaliza também as fragilidades existentes nos serviços de saúde e o despreparo para o diagnóstico precoce; as consequências da inexistência de um fluxo de atendimento que norteei a prática clínica, assim como, as falhas das políticas públicas existentes e que comprometem a assistência prestada.

Esta é a realidade da cidade cede do estudo, assim como, das microrregiões de saúde pertencentes a esta. Desta maneira, sugere-se aos gestores de saúde, a construção de um fluxograma abrangendo os níveis de atenção, o rastreamento de mulheres com fatores de risco na atenção básica e as condutas à serem adotadas como encaminhamentos para investigação e confirmação diagnóstica e/ou tratamento.

\section{REFERÊNCIAS}

A. C. CAMARGO. Manual de Condutas em Ginecologia Oncológica/A.C. Camargo Cancer Center, Departamento de Ginecologia. 2014. $2^{a}$ ed. São Paulo: FAP, 2014, p. 1-68.

ASSIS, M. M. A. et al. Dimensões teóricas e metodológicas da produção do cuidado em saúde. In. Produção do cuidado no programa saúde da família: olhares analisadores em diferentes cenários. Salvador: EDUFBA. 2010. Cap. 1. p. 13-38.

DERCHAIN, S. F. M.; FRANCO, E. D.; SARIAN, L. O. Panorama atual e perspectivas em relação ao diagnóstico precoce do câncer de ovário. Rev. Bras. Ginecol. Obstet., Rio de Janeiro, v. 31, n. 4, p. 159-163, 2009.

INSTITUTO NACIONAL DE CÂNCER JOSÉ ALENCAR GOMES DA SILVA. Inca estima quase 600 mil casos novos de câncer para 2015. INCA.gov. [2015]. Disponível em:<http://www2.inca.gov.br/wps/wcm/connect/agencianoticias/site/home/noticias/2015/inca _estima_quase_600_mil_casos_novos_de_cancer_em_2016> Acesso em: 05 dez. 2015.

Ministério da Saúde. Coordenação de Prevenção e Vigilância. Estimativa 2016: Incidência de Câncer no Brasil. Dia Nacional de Combate ao Câncer. Coordenação de Prevenção e Vigilância. INCA, 2015, p. 51.

OLIVEIRA, K. M. de. Câncer de ovário e detecção precoce: revisão bibliográfica da literatura. 2014, f. 56. Monografia (Graduação em Enfermagem) Sociedade de Educação e Cultura de Goiânia - SECG. Goiânia-GO, dez., 2014.

OLIVEIRA, M. A. C.; PEREIRA, I. C. Atributos essenciais da Atenção Primária e a Estratégia Saúde da Família. Rev. Bras. Enferm. v. 66, n. (esp), 2013, p. 158-164.

PITTA, D. R. et al. Symptoms, CA125 and HE4 for the preoperative prediction of ovarian malignancy in Brazilian women with ovarian masses. BMC Cancer, London, v. 13, n. 423, p. 1-11, Sep. 2013.

TEXEIRA. C. Os princípios dos Sistema Único de Saúde. Salvador, Bahia. p. 1-10. Jun. 2011. Disponível em: < http://www.saude.ba.gov.br/pdf/OS_PRINCIPIOS_DO_SUS.pdf > Acesso em: 03 abr. 2016.

VAUGHAN, S. et al. Rethinking ovarian cancer: recommendations for improving outcomes. Nat. Rev. Cancer., Londres, v. 23, 11, n. 10, p. 719-725, Sep. 2011. 